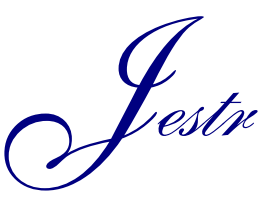

Research Article

\title{
Threshold Based Limited Feedback Bit Partitioning for Interference Channels in Multicell Systems
}

\author{
S.Balaji $^{1, *}$, P.S.Mallick ${ }^{1}$ and Gnanam Gnanagurunathan ${ }^{2}$ \\ ${ }^{1}$ School of Electrical Engineering, VIT University, Vellore - 632014, India. \\ ${ }^{2}$ Faculty of Engineering, Malaysia Campus, The University of Nottingham, Malaysia
}

Received 7 April 2016; Accepted 20 September 2016

\begin{abstract}
In this paper feedback allocation of interference channels using multi-cell coordinated beamforming is studied. By fixing the total feedback budget as constant, the proposed interference grading threshold scheme adaptively allocating the feedback bits between inter-user interference and inter-cell interference. The individual feedback bits of inter-user and inter-cell interference are calculated by employing interference grading. A new iterative algorithm is proposed to find the total residual feedback bits. The proposed feedback allocation in interfering broadcast and time varying channels are numerically evaluated and it is found that the proposed method performs comparatively better than other counterparts.
\end{abstract}

Keywords: Time Varying Channel, Interfering Broadcast channel, Interference Grading, Coordinated Beamforming, Throughput

\section{Introduction}

The interference mitigation methods in limited feedback with the advent of cooperation among Base Stations (BS) become more popular and have been studied extensively in the recent past. Recently the sum-rate performances in coordinated beamforming downlink multi-antenna techniques are analyzed in many research works. The interuser interference (IUI) and inter-cell interference (ICI) degrades the sum-rate performance in multi-antenna systems. To practically realize the achievable throughput, both the inter-cell interference and inter-user interference required to be suppressed to the needed level. The performance analysis of Random Vector Quantization (RVQ) on reducing the negative effects of these interferences in limited feedback system is studied [1]. With partial channel state information (CSI), the sum rate performance in finite rate feedback multi-antenna system is analyzed. The relation connecting the number of users, signal to noise ratio (SNR) and the number of bits for sumrate in interfering broadcast channel (IFBC) has been proposed [2]. In systems where interference becomes a key capacity limiting factor, multicell coordination with beamforming techniques proves to offer good improvement in capacity analysis. Lots of coding methods and fundamental research techniques on beamforming are also suggested for combating the interferences [3]. In addition to coordination and beamforming, the throughput performance in interference regimes with adaptive bit partitioning between ICI and IUI has been proposed in many published works. The partitioning of bits between IUI and ICI for interfering broadcast (IFBC) is analyzed in [5]. While partitioning the bits, it is shown that the bits allocation is a

*E-mail address: sbalaji@vit.ac.in

ISSN: $1791-2377$ @ 2016 Eastern Macedonia and Thrace Institute of Technology. All rights reserved. function of number of antennas and strength of the signals. While considering the interference strengths, the stronger interferences are allocated with more bits but lower interferences are allocated with lesser bits. The bit partitioning between the ICI and IUI by considering the limited feedback backhaul delay is also derived. Moreover by including the backhaul delay and interference signal strength, the BS cooperation can drastically improve the average sum-rate over the equal bit partitioning scheme [6]. In [7], using the component phase fluctuations of channel, the variation of ICI and IUI feedback in multi-cell systems with RVQ have been proposed. A comparison between multi-cell system and point to point system is made while proving that the feedback rate in multipoint system should be linearly scaled with SNR. Moreover, expressions are derived for throughput degradation in limited feedback interference channels [8]. In [9], the authors derived the average sum-rate expressions for cell edge user in multiantenna systems using RVQ and zero-forcing beamforming. It is shown that tremendous capacity improvement can be achieved with multiple antennas at the transmitter or at the receiver $[8,9]$.

A two stage feedback scheme is proposed to control the feedback rate of the user by selecting the best orthogonal users based on the channel conditions of the user [10]. To overcome the residual interference arising from ICI and IUI, an interference alignment scheme is analyzed to optimize the interference reference vector [11]. In low mobility conditions, it is proved that channels utilizing time correlation in quantization of CSI played a bigger role. This time correlation helps in determining the sum-rate under various SNR regimes [12]. Minimization of transmits power with target SNR using coordinated zero-forcing was also recently proposed [13]. Suitable expressions are derived for rate loss employing coordinated beamforming in MIMO systems by considering the time varying nature of the channel (TVC) [14-16]. The selection of optimum 
beamforming for partitioning of bits to mitigate both IUI and ICI is also discussed [17]. Consequently, the beamforming methods, coordination between users and or base stations are used to enhance the achievable throughput of the system under study, when it is severely affected by interferences.

Most of works published earlier adaptively partitioning the bits between IUI and ICI based on received interference power levels. The rate loss is characterized in these systems either by considering the channels are constant during the feedback period (IFBC) or the channels are varying in time (Time Varying Channel). In these two types of channel conditions, feedback has not been partitioned based on the significant interference level. This significant interference level categorizing is used to demarcate the area where high level of coordination among base stations is definitely required in that area to reduce the residual interferences. The objective is to reduce residual interference and the backhaul load there by adaptively partitioning the bits between IUI and ICI. The proposed threshold based adaptive allocation helps to determine whether ICI or IUI is dominant. Mathematical expressions involving interference grading threshold for both TVC and IFBC are derived for realizable throughput in coordinated zero-forcing beamforming. The interference grading threshold allows the system to utilize feedback bit of the interference which is not so high enough to ruin the sum-rate. The feedback bits are utilized by allocating to the interference which is really threatening to reduce the performance of the user. Numerical simulations are provided to demonstrate the sum-rate improvement by effectively utilizing the feedback resources of interference which has the lower strength. The derived sum -rate expression is a function of interference grading parameter besides number of antennas, feedback update period, strength of IUI, and strength of ICI.

\section{System Model}

The K-Cell Multiple Input Single Output (MISO) with finite rate feedback is considered in this work. The base stations consist of $M$ antennas and there are $N$ users. The users are served with $N$ data streams. By considering equal power allocation over $M$ antennas, the signal at the user $n$ situated in $i$-th BS is given by [5]

$$
y_{n, i}=\sqrt{\gamma_{n, i, i}} h_{n, i, i}^{H} x_{i}+\sum_{j=1, j \neq i}^{K} \sqrt{\gamma_{n, i, j}} h_{n, i, j}^{H} x_{j}+n_{n, i,}
$$

where $\gamma_{n, i, j}$ is the power of interference at the user from $j$ th $\mathrm{BS}$ at the location $n, h_{n, i, j}^{H}$ represents the interference vector with $M X 1$ dimension from $\mathrm{BS}_{\mathrm{j}}$ and transmit signal vector $x_{i}$ is of dimension $M X 1$. The channel elements are drawn from identically independently distributed complex Gaussian samples with unit variance and zero mean. The additive white Gaussian noise $n_{n, i}$, having variance of one is considered to be received by the user. If $d_{i}$ is the distance between the user and $i$-th BS, then the path loss of the signal received at the user from the $i$-th $\mathrm{BS}$ is given by $\left(1+d_{i}\right)^{-\alpha}$ where $\alpha$ represents the path loss exponent.

\section{A. Feedback Model}

It is assumed that the user in the cells has perfect knowledge of CSI of the channels received i.e. $h_{n, i, i}, h_{n, i, j}$ by exploiting the reference inter-cell orthogonal signals. From this CSI, users sends back to the serving base station, $\mathrm{BS}_{\mathrm{i}}$, a channel quality information and channel direction information of all channel links. Moreover, each user quantizes the received channel vectors directions i.e. for example, the inter-cell interference channel vector is quantized as $\tilde{h}_{n, i, j}=\frac{h_{n, i, j}}{\left|h_{n, i, j}\right|}$ where $\mathrm{j}=\{1,2 \ldots, \mathrm{K}\}$. Incorporating minimum chordal metric, distance indices for channel distribution information (CDI) of serving and interfering base stations channel vectors [8] are $\hat{h}_{n, i, j}=c_{n, i, j}^{z_{j}}$ and $z_{j}=\arg \max _{1 \leq m \leq 2}\left|c_{n, i, j}\right| c_{n, i, j}^{{ }^{m}}{ }^{H} \tilde{h}_{n, i, j} \mid$. To quantize CDI and CQI, random vector quantization (RVQ) is applied in which the codeword are distributed on a complex $M$ dimensional hypershpere. Each of the codewords is considered to be independent and isotropic. After quantization, users inform the indices $z_{j}$ to the serving BS through feedback link which is assumed as error free in this work. In our feedback analysis, channel quality information (CQI) feedback is not included in total feedback calculation and each user satisfies the total feedback constraint i.e. $K a^{C Q I}+\sum_{n=1}^{N} b_{n}=B_{\text {Total }}$, where $a^{C Q I}$ is the feedback bits for CQI of each user.

\section{B. Coordinated zero forcing feedback}

With the designed beamforming vectors, the signal received at the user [5] is written as

$$
y_{n, i}=\sqrt{\gamma_{n, i, i}} h_{n, i, i}^{H} w_{n, i} s_{n, i}+\underbrace{\sum_{n=1, m \neq l}^{N} \sqrt{\gamma_{n, i, i}} h_{n, i, m}^{H} w_{m, i} s_{m, i}}_{I U I}+\underbrace{\sum_{j=1, j \neq i}^{K} \sqrt{\gamma_{n, i, j}} h_{n, i, j}^{H} \sum_{n=1}^{N} w_{n, j} S_{n, j}}_{I C I}+n_{n, i,}
$$

In the above equation (2) $w_{n, i}$ stands for the beamforming vectors for user $(n, i)$ with size of $M X 1$ and having constraints on normalization, i.e. $\left\|w_{n, i}\right\|=1$. The variable $s_{n, i}$ denotes the data symbol for the user and $E\left(\left|s_{n, i}\right|^{2}\right)=1$. Since both quantized CDI of serving and interfering channels are known, each BS constructs the beamforming vectors $w_{n, i}$ so that inter user interference (IUI) and Inter cell interference 


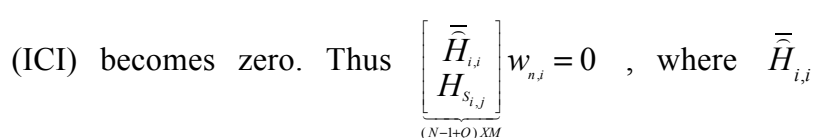
represents intra-cell complementary network channel. The channel $\overline{\hat{H}}_{i, i}$ is written as $\overline{\hat{H}}_{i, i}=\left[\hat{h}_{1, i, i,} \ldots, \hat{h}_{i-1, i, i,} \hat{h}_{l+1, i, i} \ldots, \hat{h}_{N, i, k}\right]^{H}$ and $\hat{H}_{S_{n, i}}=\left[\hat{h}_{p, k, i, \cdots}, \hat{h}_{m, j, i}\right]^{H}$ stands for the inter-cell network channel. The parameter $S_{i}$ is a set of other cell users affected by transmission of $\mathrm{BS}_{\mathrm{i}}$. The cardinality set of $S_{i}$ determines the size of the rows of $\hat{H}_{S_{l, i}}$. In finite rate feedback system, IUI and ICI are not fully eliminated and as a result of partial elimination, the rate achievable by the user is

$$
T_{n, i}^{F B}=\log _{2}\left(1+\frac{\gamma_{n, i, i}|| h_{n, i, i}||^{2}\left|\tilde{h}_{n, i, i}^{H} w_{n, i}\right|^{2}}{I U I+I C I+1}\right)
$$

In the above equation (3), the residual interference is calculated by decomposing the channel $\tilde{h}_{n, i, j}$ into two basis function. Both the basis functions are orthogonal to each other. The channel vector after quantizing the CDI is $\tilde{h}_{n, i, i}=\hat{h}_{n, i, j}\left(\cos \theta_{n, i, j}\right)+q_{n, i, j}\left(\sin \theta_{n, i, j}\right)$ and $\theta_{n, i, j}$ represents the angle between quantized channel vector and real vector. The error arising from channel quantization is taken as $q_{n, i, j}$. The rate of the users with residual ICI and IUI after the beamfoming vector designed is calculated as

$$
T_{n, i}^{F B}=\log _{2}\left(1+\frac{P_{n, i, i}\left(1+d_{i}\right)^{-\alpha}|| h_{n, i, i}||^{2}\left|\tilde{h}_{n, i, i}^{H} w_{n, i}\right|^{2}}{1+I U I+I C I}\right)
$$

The residual ICI in equation (4) is $I C I=\sum_{j=1, j \neq k}^{K} P_{n, i, j}\left(1+d_{j}\right)^{-\alpha}|| h_{n, i, j} \|^{2} \sum_{n=1}^{N} \sin ^{2} \theta_{n, i, j}\left|q_{n, i, j}^{H} w_{n, j}\right|^{2}$. Similarly the IUI in equation (4) is represented as $I U I=P_{n, i, i}\left(1+d_{i}\right)^{-\alpha}\left\|h_{n, i, i}\right\|^{2} \sin ^{2} \theta_{n, i, i} \sum_{m=1, m \neq n}^{N}\left|q_{m, i, i}^{H} w_{m, i}\right|^{2}$. The received desired signal at the user $\gamma_{n, i, i}$ is replaced with path loss as $\gamma_{n, i, i}=\frac{P_{n, i, i}}{\left(1+d_{i}\right)^{\alpha}}$ and similarly interfering signal power is replaced as $\gamma_{n, i, j}=\frac{P_{n, i, j}}{\left(1+d_{j}\right)^{\alpha}}, \gamma_{n, i, m}=\frac{P_{s, i, m}}{\left(1+d_{m}\right)^{\alpha}}$.

\section{Threshold based bit partitioning in IFBC}

The difference between the rates of perfect CSI and the rates with limited feedback CSI is termed as rate loss $\Delta T_{n, i}$ i.e. $\Delta T_{n, i}=E\left(T_{n, i}^{P C S I}-T_{n, i}^{F B}\right)$ where $T_{n, i}^{P C S I}$ represent the rate of the user when perfect Channel State Information is available. The rate with perfect $T_{n, i}^{P C S I}$ is
$T_{n, i}^{P C S I}=\log _{2}\left(1+\gamma_{n, i, i}\left|h_{n, i, i}^{H} w_{n, i}^{P}\right|^{2}\right)$

where $w_{n, i}^{P}$ is the designed beamforming vector at $\mathrm{BS}_{\mathrm{i}}$ if perfect CSI is known at the $\mathrm{BS}_{\mathrm{i}}$. The sum rate of the user in the coordinated zero-forcing beamforming system with quantized CSI and feedback through limited backhaul is

$$
E\left[T_{n, i}^{F B}\right]=E\left\{\log _{2}\left(1+\frac{P_{n, i, i}\left(1+d_{i}\right)^{-\alpha}|| h_{n, i, i}||^{2}\left|\tilde{h}_{n, i, i}^{H} w_{n, i}\right|^{2}}{I U I+I C I+1}\right)\right\}
$$

The interference from neighboring cell i.e. inter-cell interference is measured by a single parameter. The ratio between ICI and IUI is called as interference grading factor $\sigma$ in this work. The interference grading factor usually fall in the interval $[0,1)$ which is defined as ratio of IUI power to the ICI power, i.e. $\sigma=\frac{I C I}{I U I}$,

$$
\sigma=\frac{\sum_{j=1, j \neq k}^{K} P_{n, i, j}\left(1+d_{j}\right)^{-\alpha}|| h_{n, i, j} \|^{2} \sum_{n=1}^{N} \sin ^{2} \theta_{n, i, j}\left|q_{n, i, j}^{H} w_{l, j}\right|^{2}}{\left.P_{n, i, i}\left(1+d_{i}\right)^{-\alpha}|| h_{n, i, i}\right|^{2} \sin ^{2} \theta_{n, i, i} \sum_{m=1, m \neq n}^{N}\left|q_{m, i, i}^{H} w_{m, i}\right|^{2}}
$$

This grading of residual interference is to find the dominant interference which is causing significant throughput loss i.e. the IUI will be having more value rather than ICI in the regions nearer to the BS. This region where IUI is more predominant is termed as Non-Cooperative Region. In the non-cooperative region more bits are required to be allocated to the serving BS where IUI will be around $90 \%$ higher compared to ICI. Meanwhile at the cell edge, where ICI is more compared to IUI i.e. the region where cooperation becomes imperative. In this cooperative region, ICI should be given more bits compared to IUI. To categorize out of IUI and ICI which is causing significant throughput degradation, the interference grading threshold is introduced. By using this grading of interference, the interference power is measured and if the measured interference is stronger, the stronger interference is allocated higher number of bits based on the value of its interference power against the threshold $\sigma_{T}$. As soon as the graded interference is higher than or equal to the predefined threshold, the required numbers of bits are allocated to interferences based on its signal strength by using the proposed algorithm given at the end of this section. Suppose if the user is nearer to the cell edge i.e. in cooperative region, IUI more likely to be lesser than ICI. To illustrate more clearly, ICI value is equal to $50 \%$ of IUI or more (The value of ICI approximately equal to IUI at the cell edge), ICI will cause throughput degradation and higher number of bits needs to be allocated. Since in the cooperative region ICI is dominant than IUI, the algorithm compares the interference grading with significant threshold $\sigma_{\text {sig }}$, and the bits are allocated suitably between the Inter-cell interference and Inter-user interference. By substituting the interference grading parameter, the expected rate is written as 


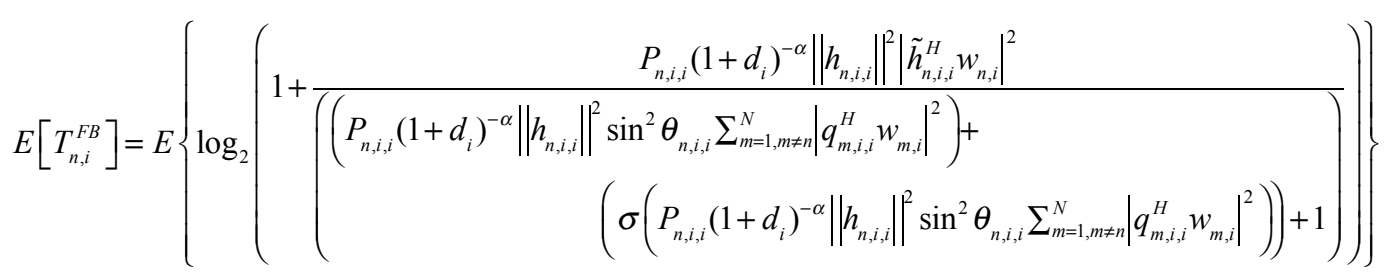

Simplifying the equation (8) yields

$$
E\left[T_{n, i}^{F B}\right]=\left\{\log _{2}\left(1+\frac{P_{n, i, i}\left(1+d_{i}\right)^{-\alpha} \| h_{n, i, i}||^{2}\left|\tilde{h}_{n, i, i}^{H} w_{n, i}\right|^{2}}{(1+\sigma)\left(P_{n, i, i}\left(1+d_{i}\right)^{-\alpha}|| h_{n, i, i} \|\left.^{2}\right|^{2} \sin ^{2} \theta_{n, i, i} \sum_{m=1, m \neq n}^{N}\left|q_{m, i, i}^{H} w_{m, i}\right|^{2}\right)+1}\right)\right\}
$$

Now using the fact that the random variables $\left\|h_{n, i, i}\right\|^{2}$, $\sin ^{2} \theta_{n, i, i}$ and $\left|q_{m, i, i}^{H} w_{m, i}\right|^{2}$ are linearly independent each other. From the bounds of quantization error [3] i.e.
$E\left(\sin ^{2} \theta_{n, i, i}\right)<2^{-\frac{b_{n}}{M-1}}$, the total bits allocated to IUI and ICI for $N$ users is $\sum_{n=1}^{N} b_{n}=B_{I}$. The variable $B_{I}$ is the total bits (Residual) allocated for both IUI and ICI interference. The average rate reduces to

$$
E\left[T_{n, i}^{F B}\right] \cong E\left\{\sum_{n=1}^{N} \log _{2}\left(\frac{P_{n, i, i}\left(1+d_{i}\right)^{-\alpha}|| h_{n, i, i}||^{2}\left|\tilde{h}_{n, i, i}^{H} w_{n, i}\right|^{2}}{1+\left[P_{n, i, j}(1+\sigma)\left(1+d_{i}\right)^{-\alpha}\left(\frac{M}{M-1}\right) 2^{-\frac{b_{n}}{M-1}}\right.}\right)\right\}
$$

For notational brevity, let us define

Subject to $1^{T} \cdot b^{n}-B_{I}=0$

$\delta_{n}=P_{n, i, i}||_{n, i, i}||^{2}\left|\tilde{h}_{n, i, i}^{H} w_{n, i}\right|^{2}$ and

$-b^{n} \leq 0$

$P_{n, i, j}(1+\sigma)\left(1+d_{i}\right)^{-\alpha}\left(\frac{M}{M-1}\right)=P_{n}$, then the above equation (10) becomes

$$
E\left[T_{n, i}^{F B}\right] \cong E\left\{\sum_{n=1}^{N} \log _{2}\left(\frac{\left(1+d_{i}\right)^{-\alpha} \delta_{n}}{1+P_{n} 2^{-\frac{b_{n}}{M-1}}}\right)\right\}
$$

The channel feedback bit allocation is now formulated mathematically as Maximize $T\left(b_{n}\right)$

Subject to

$$
K a^{C Q I}+b_{n}=B_{\text {Total }}
$$

$b_{n}:$ Non-negative number

$b_{n} \leq b_{\max }$

$b_{\max }$ is used to fix the total number of bits for CDI

where $B_{I}$ is the allocated bits to IUI and ICI. The problem appears to be convex and it can be solved by Lagrangian optimization [18]. The Lagrangian dual function is

$$
L(\lambda, v)=\inf _{b^{j} \in D}\left\{\begin{array}{l}
-\sum_{n=1}^{N} \log _{2}\left(\frac{\left(1+d_{i}\right)^{-\alpha} \delta_{n}}{1+P_{n} 2^{-\frac{b_{n}}{M-1}}}\right)+ \\
\sum_{n=1}^{N} \lambda_{n}\left(-b^{n}\right)+v\left(1^{T} \cdot b^{n}-B_{I}\right)
\end{array}\right\}
$$

The Karush-Kuhn-Tucker (KKT) conditions for Lagrangian dual function are

$-b^{n *} \leq 0,($ Primal $)$

${ }_{1}^{T} b^{n *}-B_{I}=0,(\operatorname{Pr} i m a l)$

$\lambda_{n}^{*} \geq 0,($ Dual $)$

obtained by using continuous relaxation techniques [10]. By utilizing the relaxation techniques, the problem found to be

$$
f(b)=-\sum_{n=1}^{N} \log _{2}\left(\frac{\left(1+d_{i}\right)^{-\alpha} \delta_{n}}{1+P_{n} 2^{-\frac{b_{n}}{M-1}}}\right)
$$


$-\frac{P_{n}}{(M-1)\left[2^{\frac{b_{n}}{M-1}}+P_{n}\right]}-\lambda_{n}^{*}+v^{*}=0$,

(Gradient of Lagrangian)

The solution of the above problem with slack variables $\lambda_{n}^{*}$ and $v^{*}$, found to be
$b_{n}=(M-1)\left[\log _{2}\left\{\left(P_{n}\right)\left(\frac{1}{(M-1) v}-1\right)\right\}\right]$

where $b_{n}$, is equal to $\sum_{n=1}^{N} b_{n}=B_{I}$. Substitute the $P_{n}$, the number of bits assigned to the $n$-th user is

$b_{n}=(M-1)\left[\log _{2}\left\{\left[P_{n, i, j}(1+\sigma)\left(1+d_{i}\right)^{-\alpha}\left(\frac{M}{M-1}\right)\right]\left(\frac{1}{(M-1) v}-1\right)\right\}\right]$

where $v$ is calculated using water filling algorithm. Since all $b_{n}$ to be greater than zero $\left(b_{n}>0\right)$, the value of $b_{n}$ using water filling algorithm from equation (16) is

$v=\left(\frac{1}{(M-1)}\right)\left\{\frac{\left(\prod_{n}^{N} P_{l}\right)^{\frac{1}{N}}}{\left(2^{\frac{B_{I}}{M-1}}\right)^{\frac{1}{L}}+\left(\prod_{n}^{N} P_{n}\right)^{\frac{1}{N}}}\right\}$

To calculate $B_{I}$, the following algorithm is used and the algorithm finds the value of $B_{I}$ using interference grading threshold $\sigma_{T}$. The steps of the algorithm described as

\section{Algorithm:}

Required: Define the grading threshold $\sigma_{T}, \sigma_{\text {sig }}$

1. For all MSs $i=1,2, \ldots, N$ do

2. Initialize the grading parameter $\sigma=0$

3. Find $\gamma_{n, i, i}$ for all $n$ where $n \neq m$

4. Calculate IUI for all $r_{i}$

5. for all BSs $j=1,2, \ldots, K, j \neq i$ do

6. Find $\gamma_{n, i, j}$ for all BSs $\mathrm{j}$

7. Calculate ICI for all $d_{j}$

8. Initialize $\mathrm{B}_{\mathrm{I}}=\mathrm{b}_{\max }$ for all $d_{i}$

9. For all $d_{i}, d_{j}$, find $\sigma=\frac{I C I}{I U I}$, End

10. If $\left(\sigma \geq \sigma_{T}\right.$ and $\left.\sigma<\sigma_{\text {sig }}\right)$ then

11. Calculate $B_{I}$,

12. update $b_{\max }=B_{I}$, repeat step 11 and 12 till $d_{i}=d_{j}$

13. else $\left(\sigma \geq \sigma_{\text {sig }}\right)$ then

14. Calculate $B_{I}=\left\lfloor\frac{b_{\max }}{2}+(M-1) a b s\left[\log _{2}(\sigma)\right]\right\rfloor$

15. Update $B_{I}$ for all remaining $d_{i}, d_{j}$ till $d_{i}=d_{j}$

16. End

\section{Threhold based Bit Partitioning in TVC}

The feedback bit partitioning for time varying channel with channel correlation coefficient is described in this section. In a time varying channel, the quantized channel state information should reach the BS to beamform the desired signal in order to eliminate the necessary interference before the status of the channel changes. If the CSI reaches the BS after the state of channel changes, the residual interference cannot be nullified completely. To model the time changing behavior of the channel, first order Gauss-Markov model is taken. In Gauss-Markov model, the channel time varying behavior is modeled as a delay spread $[6,14]$. The channel between the $\mathrm{n}$-th user and the corresponding $\mathrm{BS}$ is given by

$h_{n, i, i}[l]=\eta_{n, i, i} h_{n, i, i}[l-1]+\sqrt{1-\eta_{n, i, i}^{2}} w_{l, i, i}[l]$

Where $\eta_{n, i, i} \in(0,1]$ represent the fading correlation coefficient. The $h_{n, i, j}[l]$ and $w_{i, j}[l]$ denote the time varying channel vector and the beamforming vector which are defined in the equation (2). The $l$ inside the brackets represents that the channel is realized in $l$-th time instant. The feedback update period $\tau_{n, i, i}$ models the correlation coefficient for $h_{n, i, i}[l]$. The feedback period $\left\{\tau_{n, i, i} \mid n=1,2, \ldots, N\right\}$ and bits allocated to the users $\left\{b_{n, T V C} \mid n=1,2, \ldots, N\right\}$ are represented as $\tau_{n, i, i}$ and $b_{n, T V C}$, the coordinated zero forcing beamforming time varying channels sum rate is represented as

$$
E_{T V C}[T]=E\left\{\log _{2}\left(1+\frac{\left.P_{n, i, i}\left(1+d_{i}\right)^{-\alpha}|| h_{n, i, j}\left[\tau_{n, i, i}\right]\right|^{2} \mid \tilde{h}_{n, i, i}^{H} w_{n, i}\left[\left.\tau_{m, i, i}\right|^{2}\right.}{I U I+I C I+1}\right)\right\}(20)
$$

Here the desired channel $h_{n, i, i}[p]$ and $h_{n, i, j}[p]$ are time varying in nature and are modeled using Gauss Markov model. Since both the channels are time varying, the CSI needs to be updated frequently to quantify the exact throughput loss. The joint optimization of number of feedback bits with channel update period is not considered in this work and it is assumed that the feedback update period to model the correlation coefficient (time varying behavior) is taken as 2. The idea of taking the feedback update period 
equal to $2\left(\tau_{n, i, i}=2\right)$ is provide maximum correlation between two successive channel state information. By utilizing the calculation of upper bound of residual interference (both ICI and IUI) and by following the $b_{n, T V C}=(M-1)\left[\log _{2}\left\{\left(\frac{P_{n, T V C} \eta_{n, i, i}^{2\left(\tau_{n, i}-1\right)}\left(\frac{M}{M-1}\right)}{P_{n, T V C}\left(1-\eta_{n, i, i}^{2\left(\tau_{n, i}-1\right)}\right)+1}\right)\left(\frac{1}{(M-1) v_{T V C}}-1\right)\right\}\right]$ procedure used for IFBC in section 3 of this paper, the number of bits for TVC condition is derived as

The slack variable for TVC $v_{T V C}$ is calculated by using the Proposed Algorithm by taking into account the time varying nature of IUI and ICI. In TVC, the $b_{n, T V C}$ is dependent parameter of fading correlation coefficient. The fading correlation coefficient determined by the delay and the delay indirectly determines the number of feedback bits $b_{n, T V C}$. The actual number of feedback bits requirement with grading threshold and the corresponding throughput improvement is demonstrated in the next section.

\section{Numerical Results}

Through Numerical simulation, the performance of the proposed scheme is demonstrated in this section. The simulation parameters are follows. A simple two cell model is taken and the cell radius in each cell is fixed and assumed to be $500 \mathrm{~m}$. The number of antennas $M=4$, the path loss exponent $\alpha=3.8$, the number of users in each cell $N=2$ and since it is a two cell model $K=2$. The correlation coefficient $\eta_{n, i, i}$ is calculated by taking the operating frequency as $2 \mathrm{GHz}$, the duration of each frame $T_{F}$ is $5 \mathrm{~ms}$ and the speed between $n$-th user of interest and the BS is considered to be $10 \mathrm{~km} / \mathrm{h}$. The Maximum value of total feedback bits for CDI quantization per user i.e. $B_{I}$ is taken as 10 . The $P_{n, i, i}$ is set when $\gamma_{n, i, i}$ at a distance $d_{i}=500 \mathrm{~m}$ is $10 \mathrm{~dB}$.

In the Fig. 1, the bits allocation of proposed scheme is compared with the previously reported result of Namoon Lee [5]. The proposed allocation starts allocating bits to the ICI interference, if its value is significant to produce throughput loss. From the Fig. 1, the proposed scheme extends the non- cooperative region by around $25 \mathrm{~m}$ compared the previous scheme of Namoon Lee [5] if the interference grading threshold is taken as 0.3. Suppose if grading factor is assigned to a maximum value i.e. 1(In the cooperative region usually ICI will be almost equal to IUI), then the proposed scheme measures both ICI and IUI which are almost equal. Suitable bits are allocated to both ICI and IUI by the proposed algorithm. This situation is demonstrated by taking $\sigma_{\text {sig }}$ as 0.8 in the Fig. 1 . One can infer from the plot is that the proposed allocation allocates more bits to ICI rather than IUI in the cooperative region. The allocation of the proposed scheme for TVC is plotted in the Fig. 2. Since the channel is TVC, the value of ICI compared to IFBC of Fig. 1, is obtained at a greater distance. The allocation for TVC with grading threshold $\sigma_{T}=0.3$ is also plotted in Fig. 2.

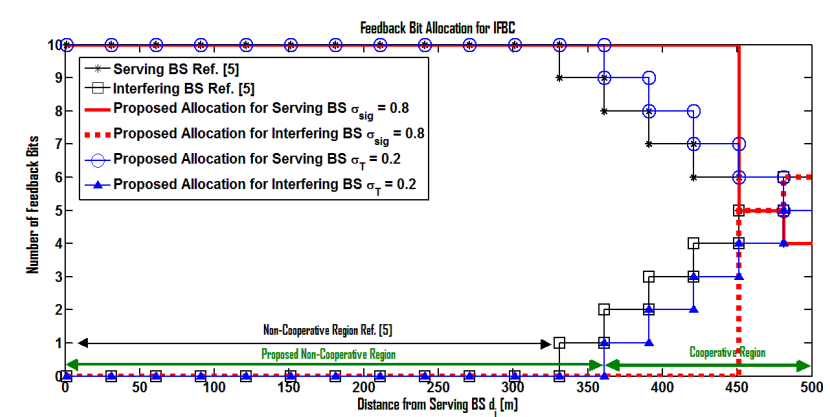

Fig. 1. IFBC Feedback Allocation for various $\sigma$

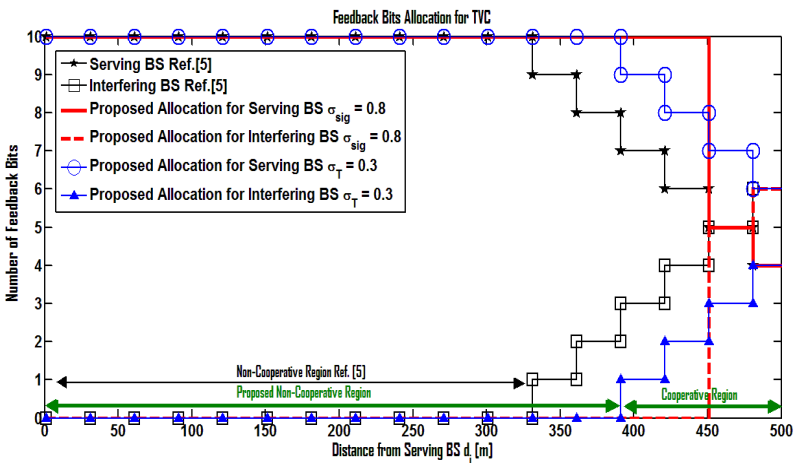

Fig. 2. TVC Feedback Allocation for various $\sigma$

The proposed IFBC and TVC throughput performance with various values of interference grading factor $\sigma$ are 0.00 to 0.3 (Non-Cooperative Region), the proposed scheme throughput is almost equal to the equal bit partitioning method. Meanwhile for $\sigma$ at cooperative region i.e. $\sigma$ of 0.4 and above, the proposed allocation outperforms the equal bit allocation. The performance improvement of about $50 \%$ in throughput is achieved compared to equal bit partitioning. These results can easily be verified by comparing the columns of Tab. 1 with respect to each $\sigma$.

The proposed bit allocation of IFBC and TVC on achievable throughput if the residual feedback bits (total) $B_{I}$ of IFBC and TVC are kept at 2 and 8 are illustrated in Fig. 3. The proposed bit allocation of IFBC scheme with $\sigma_{T}=0.3$ approximately produces $20 \%$ higher throughput in comparison with equal bit allocation scheme. The improvement reduces to $15 \%$ if one compares with previous scheme of Ref. [14]. But, when the threshold becomes $\sigma_{\text {sig }}=0.8$, the ICI is dominating, the proposed bit allocation yields slightly better result than that of both equal bit allocation and the allocation reported in Ref [5]. This is approximately equal to $1 \%-5 \%$ throughput gain compared to the other two allocation reported. In TVC also, the proposed method performs better than the equal bit and allocation of Ref [5]. The interesting inference from Fig. 3 is that as the shown in Tab. 1. One can observe that, if $\sigma$ ranges from 
total feedback bit $B_{I}$ increases i.e. if it is kept at 8 , the proposed allocation yields a greater performance compared to equal bit allocation and the previous scheme of Ref [5]. The throughput improvement of approximately 35\% with respect to equal bit allocation in both IFBC and TVC cases are observed. One can notice these results in Fig. 3 in the non-cooperative region.

Table 1. Sum -Rate for Various $\sigma$ when $B_{I}=8$

\begin{tabular}{c|c|c|c}
\hline $\begin{array}{c}\text { IUI to } \\
\text { ICI } \\
\text { Ratio } \boldsymbol{\sigma}\end{array}$ & $\begin{array}{c}\text { Equal Bit } \\
\text { Partitioning }\end{array}$ & $\begin{array}{c}\text { Proposed } \\
\text { IFBC }\end{array}$ & $\begin{array}{c}\text { Proposed } \\
\text { TVC }\end{array}$ \\
\hline 0.00 & 3.999491601 & 3.983141157 & 3.833369501 \\
0.06 & 3.858647562 & 3.944001675 & 3.764685853 \\
0.11 & 3.716107898 & 3.906035412 & 3.699593935 \\
0.165 & 3.572451785 & 3.869177816 & 3.668288031 \\
0.22 & 3.428246052 & 3.798555711 & 3.607968082 \\
0.275 & 3.284045994 & 3.764685853 & 3.578886222 \\
0.33 & 3.140397762 & 3.731713079 & 3.522725369 \\
0.385 & 2.99784205 & 3.668288031 & 3.495590752 \\
0.44 & 2.856918786 & 3.637757769 & 3.443086555 \\
0.495 & 2.718172509 & 3.607968082 & 3.392783987 \\
0.55 & 2.582158014 & 3.550481551 & 3.36840622 \\
0.605 & 2.449445725 & 3.522725369 & 3.321103462 \\
0.66 & 2.32062597 & 3.495590752 & 3.298143784 \\
0.715 & 2.196310943 & 3.46905241 & 3.253527825 \\
0.77 & 2.077132615 & 3.417670787 & 3.231841974 \\
0.825 & 1.963734249 & 3.392783987 & 3.189646044 \\
0.88 & 1.85675272 & 3.36840622 & 3.169110552 \\
0.935 & 1.756789064 & 3.321103462 & 3.129105444 \\
\hline \multicolumn{4}{|l}{}
\end{tabular}

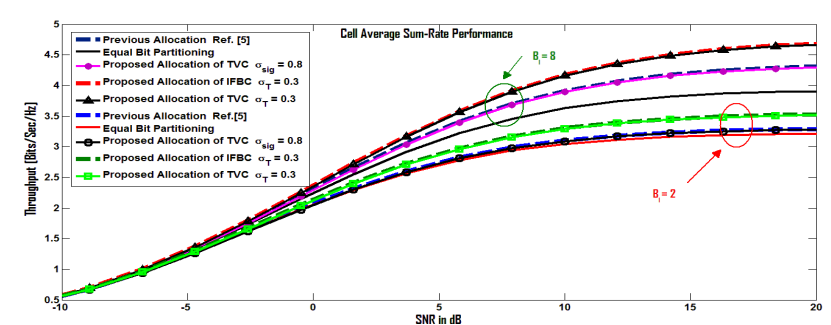

Fig. 3. Non-Cooperative Region Cell Average Sum-Rate when $B_{I}=2$ and $\mathrm{B}_{\mathrm{I}}=8$

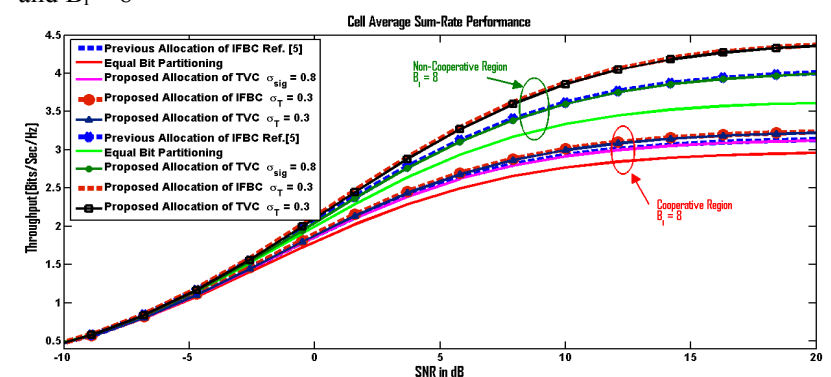

Fig. 4. Cooperative and Non-Cooperative Region Sum-Rate for Fixed $\mathrm{B}_{\mathrm{I}}=8$
By keeping the residual feedback bits $B_{I}=8$, the proposed scheme throughput results are compared between non - cooperative and cooperative regions in Fig. 4. In the non-cooperative region, the proposed scheme throughput is very much higher compared to the cooperative region because of lower ICI. Moreover, the increase in throughput in the non-cooperative region comes from the fact that higher number of feedback bits are allocated to IUI compared to ICI. This proves that ICI is not significant enough to cause throughput loss in the non - cooperative region and the feedback bits of ICI can be allocated to IUI to nullify its effect on throughput degradation.

From the Fig. 5, one can easily observe that the proposed scheme rate offset for IFBC and TVC are low in the cooperative region. Moreover, it maintains the rate loss equivalent to that of previous schemes reported in Namoon Lee [5] and Kim et al. [14] in the non-cooperative region. The interesting observation from the plot is that it validates the extension of the non-cooperative region thereby reducing the backhaul limited feedback load. It is easy to verify from the plot is that at the end of the cooperative region, the proposed scheme converges to the previous scheme. This is primarily due the same number of feedback bits are allocated by the proposed and previous schemes in high ICI region.

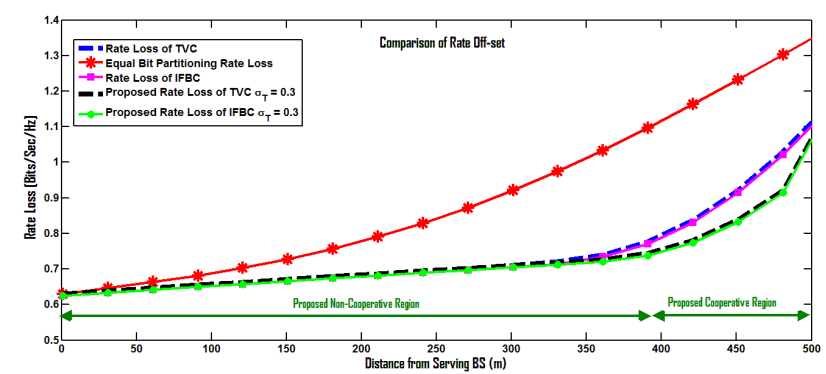

Fig. 5. Rate Off-Set with distance from serving BS

\section{Conclusion}

Adaptive bit partitioning strategy using coordinated zeroforcing beamforming for IFBC and TVC is proposed with interference grading threshold. The proposed method produces a remarkable reduction in limited feedback load for backhaul link. Unlike conventional schemes, the proposed optimization with interference grading forbids the feedback resource allocation to in-significant interferences there by clearly demarcating the cellular regions as cooperative and non-cooperative regions. Ongoing work includes the computation of feedback scaling for TVC by jointly optimizing number of bits and feedback update duration.

\section{References}

1. Chun Kin Au-Yeung, David J. Love, "On the performance of Random Vector Quantization Limited Feedback Beamforming in a MISO System", IEEE Transcations on wireless Communicationn 6 (2), pp. 458-462, Feb. 2007.

2. Taesang Yoo, Nihar Jindal, and Andrea Goldsmith, "Multi-Antenna downlink channels with limited feedback and user selection", IEEE Journal of Selected areas in communications, 25(7), pp. 1478-1491, Sep. 2007.
3. David Gesbert et al, "Multi-cell MIMO cooperative networks: A new look at interference", IEEE Journal on selected areas in communication, 28(9), pp. 1380-1408, Dec. 2010.

4. Jun Zhang and jeffrey G. Andrews, "Adaptive intercell interference cancellation in multicell wireless networks. IEEE Journal on selected areas in communications, 28(9), pp. 1455-1468, Dec. 2010.

5. Namoon Lee and Wojnae Shin, "Adaptive Feedback scheme on Kcell MISO interfering broadcast channel with limited feedback, 
IEEE Transcations on wireless communications, 10(2), pp. 401406, Feb. 2011.

6. R Bhagavatula, RW Heath, "Adaptive bit partitioning for multicell intercell interference nulling with delayed limited feedback", IEEE Transactions on Signal Processing, 59(8), pp. 3824-3836, Aug. 2011.

7. S.Balaji, R.Santhakumar, P.S.Mallick and L.Nithiyanandan. "Intercell interference nulling with bit partitioning for extended clarke's modelled multi-cell wireless networks. Australian Journal of electrical and electronics Engineering, 10(4), pp. 535-541, Dec. 2014

8. Nihar Jindal, "MIMO braodcast channels with finite rate feedback", IEEE transactions on information theory, 52 (11), pp. 5045-5060, Nov. 2006

9. S.Balaji, R.Santhakumar and P.S.Mallick. (2015). Enhancing coverage and rate of cell edge user in multi-antenna poission voronoi cells", Journal of circuits, systems and computers, Aug. 2015. DOI: $10.1142 / \mathrm{S} 0218126615501170$

10. Illsoo Sohn, Chang Soon Park, and Kwang Bok Lee, "Downlink Multiuser MIMO systems with Adaptive feedback Rate", IEEE transcations on vehicular technology, 61(3), pp. 1455-1451, Mar. 2012

11. Sungyoon Cho, Kaibin Huang, Dongku Kim and Hanbyul seo, "Interference alignment for uplink cellular systems with limited feedback", IEEE communication letters, 16 (7), pp. 960-963,Jul. 2012

12. Yuan-Pei Lin et al., "Bit allocation and statistical precoding for Correlated MIMO Channels with limited feedback", IEEE transactions on Vehicular Technology, 61(2), pp. 597-606, Feb. 2012.

13. Seongjin Kim, Deoki Kim, and Yong H. Lee, "Limited Channel feedback for coordinated beamforming under SINR requirements", IEEE international conference on communications (IEEE- ICC'13), pp. 431-435, 2013.

14. Haksoo Kim, Heejung Yu, and Yong H. Lee, "Limited Feedback for Multi-cell Zero-forcing Coordinated Beamforming in Timevarying Channels", IEEE transcations on vehicular technology, 64 (6), 2349-2360, 2015

15. Salam Akom, Robert W.Heath, jr., "Interference Coordiantion: Random clustering and Adaptive limited feedback", IEEE transcations on signal processing, 61( 7), pp. 1822-1834, Apr. 2013.

16. Kyeongyeon Kim, Taejoon Kim, David J. Love and Han Kim., "Differential feedback in codebook-Based multiuser MIMO systems in slowlying varying channels", IEEE transactions on communications, 60(2), pp. 578-588, Feb. 2012.

17. Berna Ozbek and Didier Le Ruyet, "Adaptive limited feedback links for cooperative multi-antenna multicell networks", EURASIP journal on wireless communication and networking, 2014. Doi:10.1186/1687-1499-2014-193, 2014

18. S. Boyd and L. Vandenberghe. (2004). Convex Optimization, Cambridge University Press, U.K. 